\title{
Identification of Chemotaxis Compounds in Root Exudates and Their Sensing Chemoreceptors in Plant-Growth-Promoting Rhizobacteria Bacillus amyloliquefaciens SQR9
}

\author{
Haichao Feng, ${ }^{1,2}$ Nan Zhang, ${ }^{1}$ Wenbin Du, ${ }^{3}$ Huihui Zhang, ${ }^{1}$ Yunpeng Liu, ${ }^{2}$ Ruixin Fu, ${ }^{1}$ Jiahui Shao, ${ }^{1}$ \\ Guishan Zhang, ${ }^{2}$ Qirong Shen, ${ }^{1}$ and Ruifu Zhang ${ }^{1,2, \dagger}$ \\ ${ }^{1}$ Jiangsu Provincial Key Lab for Organic Solid Waste Utilization, National Engineering Research Center for Organic-based \\ Fertilizers, Jiangsu Collaborative Innovation Center for Solid Organic Waste Resource Utilization, Nanjing Agricultural \\ University, Nanjing, 210095, P.R. China; ${ }^{2}$ Key Laboratory of Microbial Resources Collection and Preservation, Ministry of \\ Agriculture, Institute of Agricultural Resources and Regional Planning, Chinese Academy of Agricultural Sciences, Beijing \\ 100081, P.R. China; and ${ }^{3}$ State Key Laboratory of Microbial Resources, Institute of Microbiology, Chinese Academy of \\ Sciences, Beijing 100101, China
}

Accepted 19 March 2018.

\begin{abstract}
Chemotaxis-mediated response to root exudates, initiated by sensing-specific ligands through methyl-accepting chemotaxis proteins (MCP), is very important for root colonization and beneficial functions of plant-growth-promoting rhizobacteria (PGPR). Systematic identification of chemoattractants in complex root exudates and their sensing chemoreceptors in PGPR is helpful for enhancing their recruitment and colonization. In this study, 39 chemoattractants and 5 chemorepellents, including amino acids, organic acids, and sugars, were identified from 98 tested components of root exudates for the well-studied PGPR strain Bacillus amyloliquefaciens SQR9. Interestingly, mutant stain SQR9 8 8mcp, with all eight putative chemoreceptors completely deleted, lost the chemotactic responses to those 44 compounds. Gene complementation, chemotaxis assay, and isothermal titration calorimetry analysis revealed that McpA was mainly responsible for sensing organic acids and amino acids, while McpC was mostly for amino acids. These two chemoreceptors may play important roles in the rhizosphere chemotaxis of SQR9. In contrast, the B. amyloliquefaciensunique chemoreceptor McpR was specifically responsible for arginine, and residues Tyr-78, Thr-131, and Asp-162 were critical for arginine binding. This study not only deepened our
\end{abstract}

Current address for R. Zhang: College of Resources and Environmental Sciences, Nanjing Agricultural University, Nanjing, 210095, P.R. China.

${ }^{\dagger}$ Corresponding author: R. Zhang; E-mail: rfzhang@njau.edu.cn

Funding: This work was financially supported by National Natural Science Foundation of China (31672232, 31572214, 31330069, and 31500081), the National Key Basic Research Program of China (973 program and 2015CB150505), and the National Key Research and Development Program (2016YFD0200305 and 2017YFD0200805). R. Zhang and Q. Shen were also supported by the Key Projects of International Cooperation in Science and Technology Innovation (2016YFE0101100), the 111 Project (B12009), and the Priority Academic Program Development (PAPD) of Jiangsu Higher Education Institutions.

*The $\boldsymbol{e}$-Xtra logo stands for "electronic extra" and indicates that three supplementary figures and six supplementary tables are published online.

(C) 2018 The American Phytopathological Society insights into PGPR-root interaction but also provided useful information to enhance the rhizosphere chemotaxis mobility and colonization of PGPR, which will promote their application in agricultural production.

Plant-growth-promoting rhizobacteria (PGPR) are widely used in agricultural production for stimulating plant growth and suppressing soilborne diseases (Lugtenberg and Kamilova 2009), but the performance of their plant-beneficial effects depends on efficient rhizosphere colonization (Compant et al. 2010). Chemotaxis, the ability of motile bacteria to direct their movement in gradients of attractants and repellents, plays an important role during the rhizosphere colonization process of PGPR. In the rhizosphere, a nutrient gradient is formed by plant-released root exudates, which have a complex composition and include some components that may serve as chemoattractants or chemorepellents for plant-beneficial microbes (Badri and Vivanco 2009; Liu et al. 2017). Root exudatemediated chemotaxis is recognized as the initial step of the recruitment and colonization process (de Weert et al. 2002; Scharf et al. 2016; Zhang et al. 2014), which enhances the ability of bacteria to colonize the roots of diverse plant hosts (Bais et al. 2006; Berendsen et al. 2012; Caetano-Anollés et al. 1988; Scharf et al. 2016).

Bacterial chemotaxis is triggered when a stimulating molecule binds to its cognate chemoreceptor. Typical chemoreceptors are composed of a methyl-accepting (MA) domain, a cytosolic signaling domain, a HAMP (histidine kinase, adenyl cyclase, methyl-accepting chemotaxis protein [MCP] and phosphatase) linker (Aravind and Ponting 1999), and a ligand-binding domain (LBD), which is frequently located in the extracytoplasmic space and is responsible for binding extracellular compounds (Baker et al. 2006; Milburn et al. 1991). The binding of the ligand to LBD modulates the autophosphorylation of the histidine kinase CheA, which in turn transfers the phosphoryl groups to the response regulator CheY. Subsequently, the generated CheY-P permits its interaction with the flagellar motor to control cell swimming or tumbling to ultimately mediate chemotaxis (Sourjik and Wingreen 2012). 
A large number of chemoreceptors and their corresponding ligands have been characterized in different microorganisms, including Escherichia coli, Pseudomonas putida, Bacillus subtilis, and Sinorhizobium meliloti (Garrity and Ordal 1995; Sampedro et al. 2015; Sourjik 2004; Webb et al. 2017; Yssel et al. 2011). Five chemoreceptors (Tar, Tsr, Tap, Trg, and Aer) have been identified in $E$. coli, which responds to concentration gradients of amino acids, dipeptides, and sugars (Sourjik 2004). Twenty-seven MCP from the free-living environmental bacterium $P$. putida KT2440 have been characterized, while $\mathrm{McpS}$, $\mathrm{McpP}$, and McpQ are found to be chemoreceptors for tricarboxylic acid cycle intermediates, $\mathrm{C}_{2}$ and $\mathrm{C}_{3}$ carboxylic acids, and citrate/metal ion complexes, respectively (Sampedro et al. 2015). The gram-positive model bacterium B. subtilis possesses $10 \mathrm{MCP}$, including McpA, McpB, МcpC, TlpA, TlpB, TlpC, HemAT, YfmS, YvaQ, and YoaH (Mowbray and Sandgren 1998; Sourjik 2004). McpB is found to be the sole chemoreceptor for asparagine, and $\mathrm{McpC}$ can directly bind 11 amino acids (proline, threonine, glycine, serine, valine, alanine, tyrosine, isoleucine, phenylalanine, leucine, and histidine) and indirectly sense four others (lysine, arginine, methionine, and glutamine). The myoglobin-like transducer HemAT is responsible for aerotaxis (Hou et al. 2000).

Bacterial chemotaxis toward different plant-secreted molecules mediated by specific chemoreceptors in the establishment of plant-microbe interactions has been demonstrated. The chemoreceptor $\mathrm{McpU}$ in the alfalfa symbiont $S$. meliloti governed chemotaxis toward host plant root exudates following colonization through direct proline sensing (Webb et al. 2014). Similarly, the chemotaxis receptor IcpB sensing oxygen in Azorhizobium caulinodans modulated nodulation and nitrogen fixation on the stems and roots of Sesbania rostrata (Jiang et al. 2016). The $\mathrm{CtaA}, \mathrm{CtaB}$, and $\mathrm{CtaC}$ receptors in $P$. fluorescens Pf0-1 are responsible for sensing amino acids in root exudates and play an important role in root colonization (Oku et al. 2012). Recently, multiple chemotaxis receptors (including $\mathrm{McpB}, \mathrm{McpC}$, and TlpC) were found to be involved in the intact chemotaxis of B. subtilis 3610 to Arabidopsis thaliana root exudates, and this process was required for bacterial colonization (Allard-Massicotte et al. 2016).

However, the chemical composition of root exudates is extremely complex, and the chemotaxis behavior of PGPR to root exudates is an integrative effect of all root-secreted chemoattractants and chemorepellents. Systematic identification of chemoattractants and chemorepellents in root exudates and elucidation of how these various root-secreted compounds are sensed by multiple chemoreceptors of a PGPR strain could give us a comprehensive understanding of PGPR chemotaxis in the rhizosphere. In this study, B. amyloliquefaciens SQR9, a well-studied and commercially widely used PGPR strain, which showed chemotaxis toward root exudates and formed obvious biofilms on plant roots (Liu et al. 2017; Weng et al. 2013), was used to explore the chemotaxis compounds in root exudates. The objectives of this study were to (i) detect the chemoattractants (or chemorepellents) in root exudates sensed by SQR9 and (ii) identify the SQR9 chemoreceptors involved that were responsible for these detected compounds. These results will provide guidelines to enhance the rhizosphere colonization and beneficial function of PGPR in agricultural production.

\section{RESULTS}

\section{Identification of chemotactic compounds for PGPR strain B. amyloliquefaciens SQR9 in root exudates.}

B. amyloliquefaciens SQR9 (Table 1) was isolated from the cucumber rhizosphere and showed positive chemotactic responses to root exudates (Liu et al. 2017; Weng et al. 2013). Also, the transcription of its chemotaxis and motility genes was upregulated by root exudates (Zhang et al. 2015). In this study, to have a comprehensive understanding of the chemoattractants in root exudates, 98 commercially available chemical compounds were selected based on results of high-throughput analysis of cucumber root exudates conducted by Liu et al. (2017); this analysis included sugars, sugar alcohols, sugar

Table 1. Bacterial strains and plasmids used in this study

\begin{tabular}{|c|c|c|}
\hline Strains or plasmid & Characteristics $^{\mathbf{a}}$ & Source or reference \\
\hline \multicolumn{3}{|l|}{ Plasmids } \\
\hline pTPC & pMD19-T harboring the PC cassette & Zhou et al. 2017 \\
\hline pNW33N & $\mathrm{Cm}^{\mathrm{R}}$, Escherichia coli-Bacillus shuttle vector & Zhou et al. 2017 \\
\hline p7S6 & $\mathrm{Spc}^{\mathrm{R}}$, pMD18-T ligated with $s p c$ gene & Yan et al. 2008 \\
\hline pET-28a & Expression vector & Novagen \\
\hline pET-28a-McpALBD & pET28a derivative for expression of McpALBD & This work \\
\hline pET-28a-McpBLBD & pET28a derivative for expression of McpBLBD & This work \\
\hline pET-28a-McpCLBD & pET28a derivative for expression of McpCLBD & This work \\
\hline pET-28a-TlpALBD & pET28a derivative for expression of TlpALBD & This work \\
\hline pET-28a-TlpBLBD & pET28a derivative for expression of TlpBLBD & This work \\
\hline pET-28a-McpRLBD & pET28a derivative for expression of McpRLBD & This work \\
\hline \multicolumn{3}{|c|}{ Strains } \\
\hline \multicolumn{3}{|c|}{ Bacillus amyloliquefaciens } \\
\hline SQR9 & Wild-type isolate & Cao et al. 2011 \\
\hline $\mathrm{SQR} 9 \Delta 8 m c p$ & Deletion in all eight putative $m c p$ genes & This work \\
\hline SQR9 $\Delta 8 m c p / m c p A$ & $C m^{R}$, SQR9 $\triangle 8 m c p$ with $m c p A$ gene & This work \\
\hline SQR $9 \Delta 8 m c p / m c p B$ & $C m^{R}, \mathrm{SQR} 9 \Delta 8 m c p$ with $m c p B$ gene & This work \\
\hline $\mathrm{SQR} 9 \Delta 8 m c p / m c p C$ & $S p c^{R}, \mathrm{SQR} 9 \Delta 8 m c p$ with $m c p C$ gene & This work \\
\hline SQR9 $\triangle 8 m c p / t l p A$ & $C m^{R}, \mathrm{SQR} 9 \Delta 8 m c p$ with $t l p A$ gene & This work \\
\hline SQR9 $\triangle 8 m c p / t l p B$ & $C m^{R}, \mathrm{SQR} 9 \Delta 8 m c p$ with $t l p B$ gene & This work \\
\hline SQR9 $48 m c p / m c p R$ & $S p c^{R}, \mathrm{SQR} 9 \Delta 8 m c p$ with $m c p R$ gene & This work \\
\hline SQR9 $\triangle 8 m c p / h e m A T$ & $C m^{R}$, SQR9 $\triangle 8 m c p$ with hemAT gene & This work \\
\hline SQR9 $48 m c p / y f m S$ & $C m^{R}$, SQR9 $\triangle 8 m c p$ with $y f m S$ gene & This work \\
\hline \multicolumn{3}{|l|}{ E. coli } \\
\hline BL21 (DE3) & 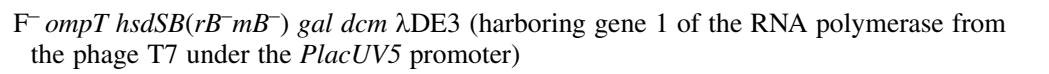 & Invitrogen \\
\hline Top 10 & 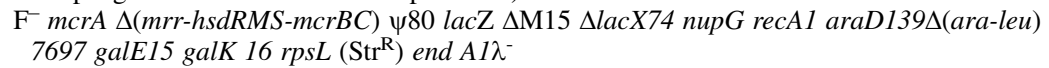 & Invitrogen \\
\hline
\end{tabular}

${ }^{\text {a }} \mathrm{Cm}^{R}=$ chloramphenicol resistance, $\mathrm{Spc}^{R}=$ spectinomycin resistance, and $\mathrm{Str}^{\mathrm{R}}=$ streptomycin resistance. 
acids, amines, amino acids, fatty acids, organic acids, and others (Table 2). These chemicals were tested individually for the chemotactic response of SQR9 using a simple and reusable microfluidic SlipChip device, as described by Shen et al. (2014). Results showed that SQR9 has a chemotactic response to 44 of these 98 tested compounds (Table 2; Supplementary Table S1). Among them, 23 compounds were strong chemoattractants, with a chemotaxis index $\left(I_{30}\right)$ of more than 0.8 (Table 2). Sixteen compounds were demonstrated as medium chemoattractants, with an $I_{30}$ between 0.6 and 0.8 (Table 2). Additionally, few root exudate compounds (DL-dithiothreitol, hydroxycarbamate, pentadecanoic acid, salicylic acid, and sodium decanoate) were characterized as repellents for SQR9, with an $I_{30}$ less than 0.4 . These results indicated that most of these responsive components served as chemoattractants, which may explain the general positive chemotactic response of SQR9 to total root exudates, as reported previously (Liu et al. 2017; Weng et al. 2013). However, more than half (54 of 98) of the tested components did not have any chemotaxis effect on SQR9.

\section{Detection of MCP in B. amyloliquefaciens SQR9.}

Chemoattractants or chemorepellents are sensed by bacterial chemoreceptors. To elucidate how these identified rootsecreted compounds were recognized by SQR9, the potential MCP genes were predicted according to the existing of MA domain (Alexander and Zhulin 2007) by using the wholegenome sequence of SQR9 (National Center for Biotechnology Information [NCBI] accession number CP006890) (Zhang et al. 2015). Eight potential MCP-encoding genes were detected, which included mсpA (V529_30840), тсрB (V529_30860), $m c p C$ (V529_13280), tlpA (V529_30850), tlpB (V529_30830), V529_10510, hemAT (V529_09980), and yfmS (V529_06960) (Fig. 1A). Compared with the well-studied B. subtilis strains 168 and OI1085, seven predicated MCP in SQR9 were conserved, and a unique chemoreceptor gene, V529_10510 (designated as $m c p R$ ), was found to only exist in SQR9 (Fig. 1B). B. amyloliquefaciens $\mathrm{SQR} 9$ appeared to have fewer MCP, because three chemoreceptors (TlpC, YoaH, and YvaQ) of strain 168 were found to be absent in SQR9. According to the report of Yssel et al. (2011), the similarity of homologous chemotaxis proteins between B. subtilis and B. amyloliquefaciens was only about $70 \%$. These findings suggested that, though they are close-related organisms, the specific functions of MCP in B. amyloliquefaciens are quite different from those in $B$. subtilis.

The domain architecture analysis of the eight predicated MCP based on the SMART database (Schultz et al. 1998) indicated that, except for HemAT and YfmS, the predicted extracellular domain existed in all six other MCP, and five of them (excluding McpR) had a conserved LBD for sensing environmental signals (Fig. 1A). The LBD of McpA, McpB, McpC, TlpA, and TlpB in B. amyloliquefaciens SQR9 contain dCache domains and belong to cluster II based on their molecular sizes (239, 246, 242, 239, and 245 amino acids, respectively). However, the LBD of McpR contains CHASE3 and belongs to cluster I (138 amino acids).

BLAST analysis based on the McpR amino acid sequence retrieved 67 alignments that shared high similarity (identity $>51 \%$, including McpR itself) in NCBI (Supplementary Table $\mathrm{S} 2$ ), most of which originated from $B$. amyloliquefaciens and $B$. velezensis (30 alignments for each). Additionally, sequences from B. nakamurai (two alignments), B. subtilis (one alignment), and Bacillus sp. (three alignments) were also obtained. $B$. amyloliquefaciens and $B$. velezensis are considered to be a taxonomic unit above the species level and form "operational group B. amyloliquefaciens" within the B. subtilis species complex (Fan et al. 2017). More than $50 \%$ of B. amyloliquefaciens
(55.4\%) and B. velezensis (53.6\%) strains contain McpR, whereas only $12.5 \%$ B. nakamurai strains and $1.8 \%$ B. subtilis strains contain McpR. These analyses indicate that McpR may be a unique chemoreceptor of B. amyloliquefaciens.

SQR9 8 mcp, deficient in all eight putative mcp genes, completely lost chemotactic reaction to 44 compounds.

To elucidate the roles of these detected MCP in SQR9's chemotactic response to these root-secreted chemoattractants

Table 2. Ranking and summary of the chemotaxis index $\left(I_{30}\right)$ of SQR9 in response to the 98 targeted compounds

\begin{tabular}{|c|c|c|c|c|}
\hline \multirow[b]{2}{*}{ Ranking (n), substances ${ }^{a}$} & \multicolumn{2}{|c|}{ SQR9 } & \multicolumn{2}{|c|}{ SQR9D8mcp } \\
\hline & $I_{30}^{\mathrm{b}, \mathrm{c}}$ & $\begin{array}{c}\text { Standard } \\
\text { errorc }^{c}\end{array}$ & $I_{30}^{\mathrm{b}, \mathrm{c}}$ & $\begin{array}{c}\text { Standard } \\
\text { error }^{\mathrm{c}}\end{array}$ \\
\hline \multicolumn{5}{|l|}{ Approximately $0.8-1.0$ (23) } \\
\hline Valine $^{\mathrm{d}}$ & 0.99 & 0.0026 & 0.48 & 0.052 \\
\hline Glutamine & 0.99 & 0.0017 & 0.51 & 0.047 \\
\hline Serine & 0.99 & 0.0018 & 0.53 & 0.049 \\
\hline Threonine & 0.98 & 0.0024 & 0.49 & 0.036 \\
\hline Succinic acid & 0.98 & 0.0030 & 0.43 & 0.049 \\
\hline Cystine & 0.98 & 0.0034 & 0.47 & 0.029 \\
\hline DL-malic acid & 0.98 & 0.0038 & 0.53 & 0.057 \\
\hline Gluconic acid & 0.97 & 0.0035 & 0.49 & 0.039 \\
\hline Asparagine & 0.97 & 0.0071 & 0.47 & 0.039 \\
\hline Citric acid & 0.95 & 0.016 & 0.47 & 0.019 \\
\hline D-maltose & 0.94 & 0.0048 & 0.54 & 0.030 \\
\hline Methionine & 0.93 & 0.016 & 0.47 & 0.032 \\
\hline Alanine & 0.93 & 0.014 & 0.55 & 0.040 \\
\hline Leucine & 0.93 & 0.036 & 0.45 & 0.043 \\
\hline Arginine & 0.91 & 0.027 & 0.55 & 0.029 \\
\hline Histidine & 0.90 & 0.022 & 0.47 & 0.064 \\
\hline Isoleucine & 0.90 & 0.0089 & 0.53 & 0.029 \\
\hline Glycine & 0.88 & 0.031 & 0.58 & 0.061 \\
\hline Fumaric acid & 0.86 & 0.019 & 0.57 & 0.027 \\
\hline Proline & 0.81 & 0.035 & 0.57 & 0.062 \\
\hline Dulcitol & 0.81 & 0.028 & 0.48 & 0.075 \\
\hline Lysine & 0.81 & 0.015 & 0.51 & 0.038 \\
\hline D-mannose & 0.81 & 0.026 & 0.50 & 0.062 \\
\hline \multicolumn{5}{|l|}{ Approximately $0.6-0.8$ (16) } \\
\hline Adipic acid & 0.79 & 0.022 & 0.52 & 0.026 \\
\hline Glutamic acid & 0.78 & 0.019 & 0.52 & 0.046 \\
\hline Phthalic acid & 0.77 & 0.019 & 0.48 & 0.033 \\
\hline Aspartic acid & 0.76 & 0.078 & 0.51 & 0.058 \\
\hline Fructose & 0.74 & 0.033 & 0.52 & 0.039 \\
\hline Tryptophan & 0.73 & 0.025 & 0.50 & 0.021 \\
\hline Phenylalanine & 0.73 & 0.038 & 0.50 & 0.029 \\
\hline DL-glyceric acid & 0.72 & 0.025 & 0.50 & 0.037 \\
\hline Dehydroascorbic acid & 0.71 & 0.048 & 0.50 & 0.037 \\
\hline Oxalic acid & 0.70 & 0.030 & 0.45 & 0.054 \\
\hline Ribitol & 0.69 & 0.038 & 0.50 & 0.055 \\
\hline Tyrosine & 0.67 & 0.025 & 0.49 & 0.023 \\
\hline $\mathrm{D}(+)$-Ribose & 0.63 & 0.033 & 0.45 & 0.028 \\
\hline Inosine & 0.63 & 0.050 & 0.50 & 0.058 \\
\hline 3-Hydroxypropionic acid & 0.62 & 0.042 & 0.49 & 0.038 \\
\hline D-(+)-fucose & 0.61 & 0.029 & 0.48 & 0.049 \\
\hline Approximately $0.4-0.6(54)^{\mathrm{e}}$ & $\ldots$ & $\ldots$ & $\ldots$ & $\ldots$ \\
\hline \multicolumn{5}{|l|}{ Approximately $0.0-0.4$ (5) } \\
\hline DL-dithiothreitol & 0.31 & 0.080 & 0.49 & 0.041 \\
\hline Salicylic acid & 0.29 & 0.023 & 0.51 & 0.034 \\
\hline Hydroxycarbamate & 0.29 & 0.041 & 0.51 & 0.045 \\
\hline Pentadecanoic acid & 0.20 & 0.016 & 0.49 & 0.043 \\
\hline Sodium decanoate & 0.16 & 0.028 & 0.50 & 0.061 \\
\hline
\end{tabular}

${ }^{\text {a }}$ Compounds were used at a concentration of $1 \mathrm{mM}$.

b When the $I_{30}$ was between 0.4 and 0.6 , this was considered to be no chemotaxis response. When the $I_{30}$ was less than 0.4 , the compound was considered to be repellent. When the $I_{30}$ was more than 0.6 , the compound was considered to be an attractant.

${ }^{c}$ Data for each compound are the means for the 13 biological replicates. Error bars represent the standard error from the mean.

d All 20 amino acids are L-amino acids.

e Data provided in Supplementary Table S1. 
and chemorepellents, all eight MCP-encoding genes were deleted from the SQR9 genome to obtain the strain SQR9 $88 m c p$. Chemotaxis experiments of SQR9 $\Delta 8 m c p$ with the 44 identified chemoattractants and chemorepellents showed that SQR9 $\Delta 8 m c p$ completely lost the chemotaxis ability to the 44 compounds (Table 2). These results suggest that these eight predicted genes encode the major functional MCP, which are critical for SQR9's chemotactic response to root-secreted chemoattractants and chemorepellents.

\section{McpA is a major chemoreceptor of SQR9} for a broad range of chemoattractants.

To determine the chemoreceptor responsible for these rootsecreted chemoattractants and chemorepellents, each of the eight MCP-encoding genes was individually complemented in $\mathrm{SQR} 9 \Delta 8 m c p$, and the chemotaxis of the complementary strains to these 44 compounds were tested. Expression of McpA (SQR9 $\Delta 8 m c p / m c p A)$ significantly restored chemotaxis to 20 compounds (Fig. 2A), including five amino acids (aspartic acid, glutamic acid, isoleucine, lysine, and tyrosine), 10 organic acids (citric acid, malic acid, oxalic acid, fumaric acid, succinic acid, phthalic acid, adipic acid, dehydroascorbic acid, glyceric acid, and 3-hydroxypropionic acid), and five other compounds (hydroxycarbamate, mannose, ribose, fucose, and ribitol). SQR9 $48 m c p / m c p A$ also partially restored the chemotactic reaction to sodium decanoate, serine, gluconic acid, and fructose, indicating that McpA probably participated in but did not dominate the chemotaxis to these compounds (Table 3; Supplementary Table S3). These results suggest that McpA senses a broad range of root-secreted compounds and is a major chemoreceptor of SQR9.

To further verify the above chemotaxis assay, the aminoterminal sensing domain of McpA (Ala33-Pro278) was expressed and purified (Supplementary Fig. S1), and its binding of citric acid and aspartic acid (representing the McpA sensed organic acids and amino acids, respectively) was investigated using isothermal titration calorimetry (ITC) analysis. The ITC results indicated that the LBD of McpA directly bound the two compounds in vitro and that the binding reactions were exothermic, with an apparent dissociation constant $\left(K_{D}\right)$ value of $0.39 \pm 0.15$ and $0.24 \pm 0.16 \mu \mathrm{M}$ for citric acid and aspartic acid, respectively. The enthalpy change $(\Delta H)$ values for the two ligands were calculated to be $-14.34 \pm 0.47$ and $-10.37 \pm 0.48 \mathrm{kcal}$ $\mathrm{mol}^{-1}$, respectively (Fig. 2B).

\section{Other chemoreceptors of SQR9 were responsible for fewer compounds.}

Compared with McpA, other chemoreceptors of SQR9 showed a relatively narrow binding ligand range (Fig. 3A). Complementation of hemAT and yfmS (SQR9 $8 m c p / h e m A T$ and SQR9 $\triangle 8 m c p / y f m S$ ) did not have any chemotactic effect regarding these tested compounds (Supplementary Table S4). Thus, these two genes were not involved in the chemotaxis of SQR9 to the root-secreted compounds, which may be due to their lack of extracellular region. For the 44 tested compounds, McpB was only responsible for four amino acid attractants (glycine, tryptophan, asparagine, and glutamine), as well as two chemorepellents (salicylic acid and sodium decanoate) (Fig. $3 \mathrm{~A})$. McpC was mostly responsible for amino acids, because nine of the SQR9 $\Delta 8 m c p / m c p C$-restored chemoattractants were amino acids (valine, alanine, threonine, proline, serine, leucine, cystine, methionine, and histidine) (Fig. 3A). TlpA only showed a response to a chemorepellent, DL-dithiothreitol, while TlpB responded to five chemoattractants (phenylalanine, maltose, fructose, dulcitol, and inosine), mostly sugars, and a potential chemorepellent of pentadecanoic acid verified by ITC subsequently (Fig. 3A).

ITC analysis of the purified LBD of McpB (Glu33-Pro278), McpC (Lys33-Met274), TlpA (Ala33-Pro278), and TlpB (Ala33-Met282) with certain compounds was conducted to verify the results of the chemotaxis experiments of complementary strains (Fig. 3B). The $K_{D}$ and $\Delta H$ values for McpBLBD and sodium decanoate binding were $3.44 \pm 2.17 \mu \mathrm{M}$ and $-1.30 \pm$ $0.18 \mathrm{kcal} \mathrm{mol}^{-1}$, respectively; the $K_{D}$ values for binding of
A YfmS

HemAT

McpR

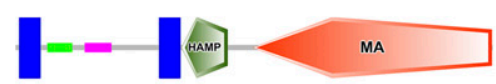

$\mathrm{McpC}$

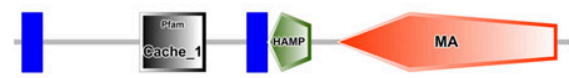

TlpB

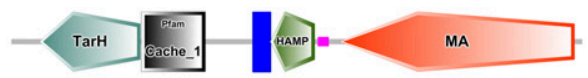

McpA

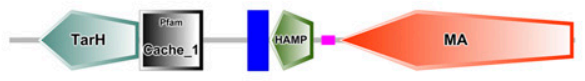

TIpA

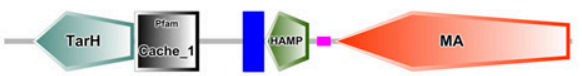

McpB

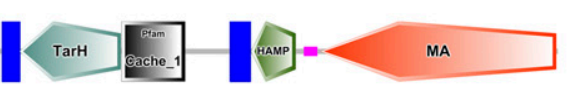

B

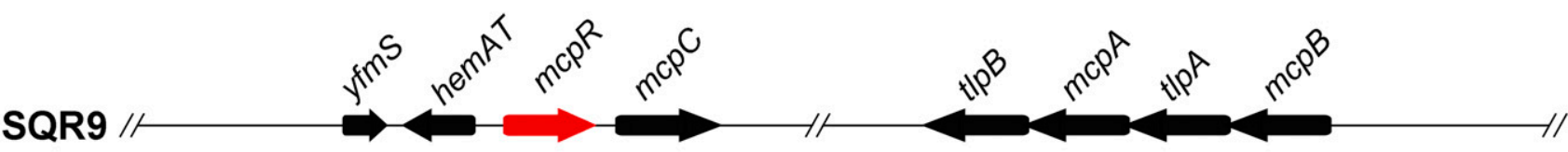

168

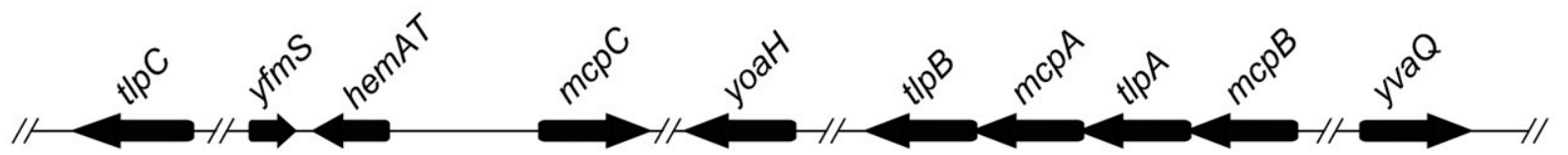

Fig. 1. Eight potential methyl-accepting chemotaxis proteins (MCP) in SQR9. A, Domain architectures of the eight MCP in the SQR9 were analyzed according to the SMART database (Schultz et al. 1998). B, Comparison of the MCP in Bacillus amyloliquefaciens SQR9 and B. subtilis 168. There were 8 MCP genes in the B. amyloliquefaciens SQR9 genome and 10 MCP genes in B. subtilis 168. The double-slash stands for a substantial distance between the adjacent genes. 
McpCLBD with leucine and proline were $3.64 \pm 0.78$ and $3.62 \pm 0.78 \mu \mathrm{M}$, respectively; and the $\Delta H$ values were calculated to be $-81.05 \pm 7.01$ and $-85.63 \pm 7.48 \mathrm{kcal} \mathrm{mol}^{-1}$, respectively. The $K_{D}$ and $\Delta H$ values for the binding of TlpBLBD with phenylalanine and pentadecanoic acid were $3.17 \pm 0.89$ and $3.03 \pm 0.66 \mu \mathrm{M}$, and $-32.24 \pm 3.39$ and $-25.68 \pm 1.86 \mathrm{kcal} \mathrm{mol}^{-1}$, respectively.

The unique chemoreceptor McpR in B. amyloliquefaciens specifically mediates chemotaxis to arginine.

McpR is a unique chemoreceptor of B. amyloliquefaciens. Complementation of McpR (SQR9 $\Delta 8 m c p / m c p R$ ) fully restored the chemotactic response to arginine but not to the other 19 amino acids (Supplementary Table S5). Furthermore, the sensing of arginine by McpR was specific in SQR9. The other seven chemoreceptor gene complementary strains did not show chemotactic response to arginine (Fig. 4A), and $\mathrm{SQR} 9 \Delta 8 m c p / m c p R$ hardly showed any chemotactic response to the other tested compounds, except a slight positive chemotaxis toward succinic acid and fucose with very low $I_{30}$ values compared with the other seven chemoreceptor gene complementary strains, which suggested that McpR may partially participate in the response to succinic acid and fucose (data not shown). The expressed and purified amino-terminal sensing domain of McpR (Ser35-Gln165) directly bound to arginine in vitro in the ITC analysis, with $K_{D}$ and $\Delta H$ values of $8.38 \pm 1.41 \mu \mathrm{M}$ and $40.36 \pm$ $1.91 \mathrm{kcal} \mathrm{mol}^{-1}$, respectively (Fig. 4B).

To further uncover the potential arginine-binding residues in the McpRLBD the sequences of the reported chemoreceptors for arginine were obtained. The McpC in B. subtilis OI1085 was reported to bind L-Arg with significantly higher affinity $\left(K_{D}\right.$ of approximately $\left.100 \mu \mathrm{M}\right)$ (Glekas et al. 2012), while McpB was also involved in chemotaxis to L-Arg (Yssel et al. 2011). Both PctALBD and PctBLBD of $P$. aeruginosa PAO1 could bind L-Arg with a $K_{D}$ of 1 and $60 \mu \mathrm{M}$, respectively, and PctA is the major receptor for L-Arg (Rico-Jiménez et al.

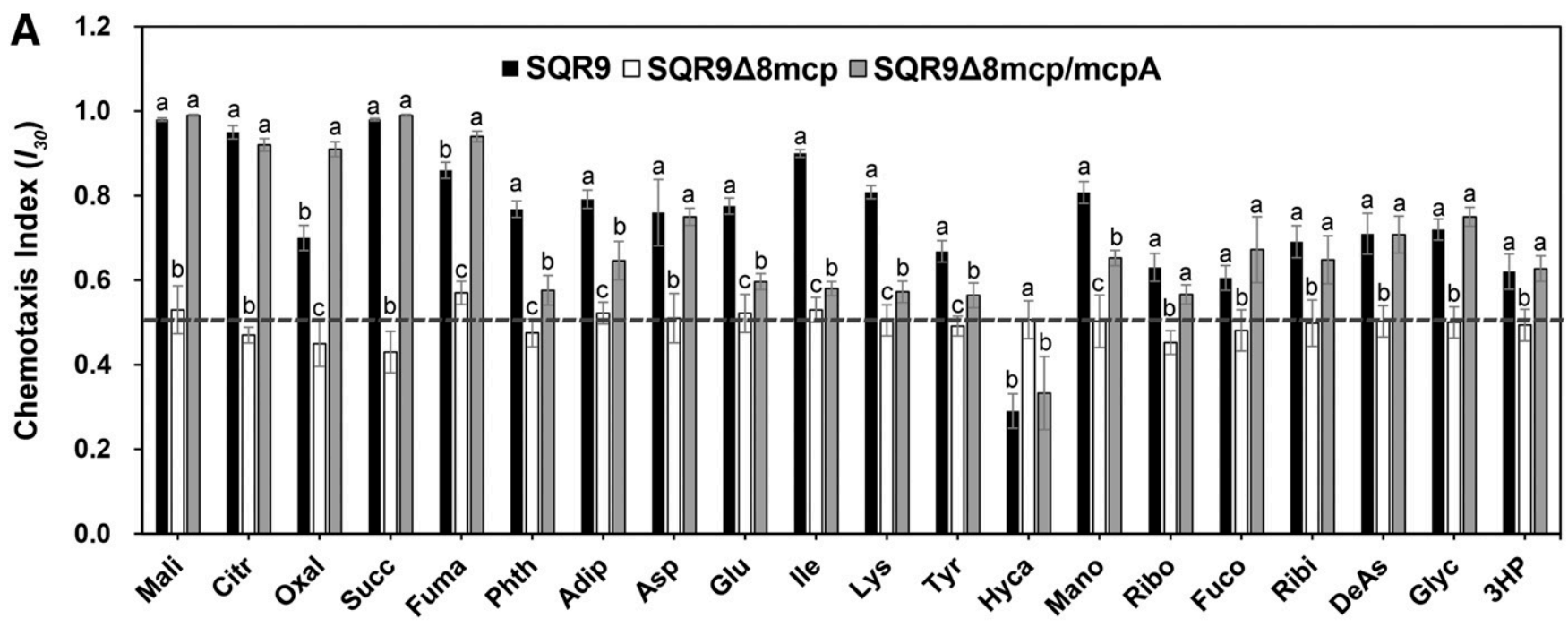

B
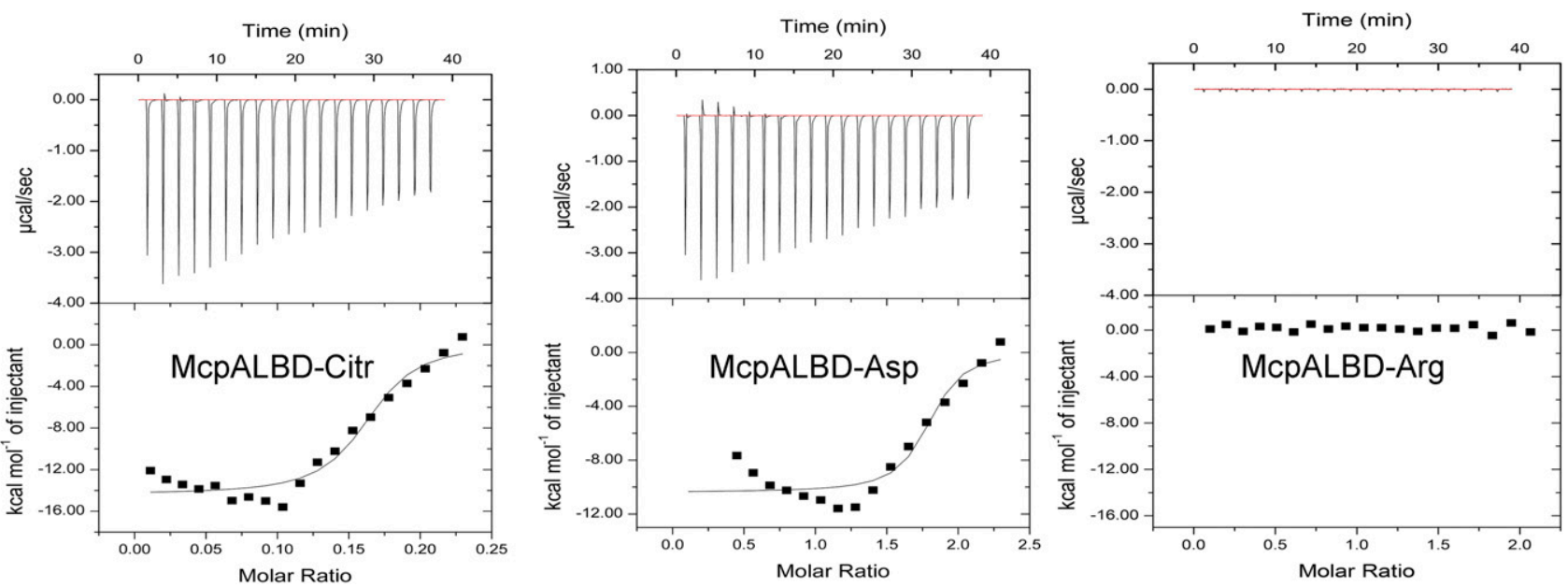

Fig. 2. Chemotaxis activity mediated by the McpA chemoreceptor. A, Chemotactic responses of SQR9, SQR9 $8 m c p$, and SQR9 $8 m c p / m c p A$ to 20 compounds. The black dotted line represents a chemotaxis index of 0.5 . The concentration of all the compounds was $1 \mathrm{mM}$. Data for each strain are the means of 13 biological replicates. Error bars represent the standard error of these 13 biological replicates. Different letters above the bars indicate significant differences between the three strains for each compound $(P \leq 0.05)$. B, Titration of $18 \mu \mathrm{M}$ McpALBD with $200 \mu \mathrm{M}$ citric acid, aspartic acid, and arginine (as a negative control). Upper panels contain the titration raw data and the lower panels show the integrated and dilution-corrected peak areas of the raw data. Data were fitted with the "one binding site model" of the MicroCal version of ORIGIN. Abbreviations: Mali = malic acid, Citr = citric acid, Oxal = oxalic acid, Succ = Succinic acid, Fuma $=$ Fumaric acid, Phth = phthalic acid, Adip = adipic acid, Asp = aspartic acid, Glu = glutamic acid, Ile $=$ isoleucine, Lys $=$ lysine, Tyr $=$ tyrosine, Hyca $=$ hydroxycarbamate, Mano $=$ mannose, Ribo $=$ ribose, Fuco $=$ fucose, Ribi $=$ ribitol, DeAs $=$ dehydroascorbic acid, Glyc $=$ glyceric acid, and $3 \mathrm{HP}=$ 3-hydroxypropionic acid. 
2013). Amino acid sequence alignment (using ClustalW and MAFFT) of the five arginine-receptor LBD revealed that residues Tyr-78, Asp-162, and Thr-131 may be crucial for arginine binding in McpR of SQR9 (Supplementary Fig. S2). For confirmation, these three residues were individually substituted by glycine, which cannot form hydrogen bonds with small ligands and is the smallest amino acid, to avoid causing possible structure disruption in the putative binding pockets. Chemotaxis analysis demonstrates that the three SQR9 $\Delta 8 m c p / m c p R$ strains with single amino-acid mutations of $m c p R$ showed significantly decreased chemotaxis to arginine compared with wild-type $m c p R$ (Fig. 5). Taken together, these results showed that the residues Tyr-78, Thr-131, and Asp-162 are the amino acid residues in McpR that are involved in arginine binding.

\section{DISCUSSION}

B. amyloliquefaciens SQR9 is a plant-beneficial rhizobacterium with a strong root colonization capability and positive chemotaxis toward cucumber root exudates (Weng et al. 2013), whose composition has been identified through gas chromatography-mass spectrometry (Liu et al. 2017). In this study, we show that PGPR strain B. amyloliquefaciens SQR9 chemotactically responded to nearly half of the tested root exudate components (44 of the 98 compounds); 39 of them were chemoattractants, while only 5 compounds served as chemorepellents. Amino acids, organic acids, and sugars were the major chemoattractant compounds and accounted for 45,32 , and $11 \%$ of the identified chemoattractants, respectively. The chemoreceptors McpA and McpC, which are mainly responsible for sensing amino acids and organic acid chemoattractants, may play critical roles in the chemotaxis mobility of SQR9 to the plant root (Fig. 6).
Generally, root exudates were divided into two different classes of compounds: the low-molecular-weight compounds, including a variety of amino acids, organic acids, sugars, and secondary metabolites, and high-molecular-weight compounds, including proteins and polysaccharides (Badri and Vivanco 2009; Liu et al. 2017). Thus far, most of the identified chemoattractants of root exudates for rhizobacteria were lowmolecular-weight compounds, which are carbon and energy resources in the environment. Additionally, chemotaxis is believed to assist rhizobacteria in moving to areas suitable for growth (Jiang et al. 2016; Lacal et al. 2010; Porter et al. 2011; Szurmant and Ordal 2004).

As an important microorganism that is closely related to B. amyloliquefaciens, B. subtilis OI1085 showed a chemotaxis response to all $20 \mathrm{~L}$-amino acids, which were predominantly mediated by McpC (supports chemotaxis to 17 and directly binds to 11 amino acids) and $\mathrm{McpB}$ (supports chemotaxis to 4 amino acids) (Hanlon and Ordal 1994). Here, we found that the sensing of amino acids in B. amyloliquefaciens SQR9 was dispersed among different chemoreceptors, which is significantly different from B. subtilis OI1085. In SQR9, McpA, $\mathrm{McpB}, \mathrm{McpC}, \mathrm{TlpB}$, and McpR responded to 6, 7, 9, 2, and 1 amino acids, respectively (Table 3 ). Interestingly, the extracellular regions of the chemoreceptors McpA, McpB, and McpC of SQR9 were similar to the chemoreceptors McpB and McpC of B. subtilis, which were predicted to possess double PDC sensor domains (Glekas et al. 2010, 2012; Rico-Jiménez et al. 2013; Sampedro et al. 2015). McpC was the dominant amino acid sensor in SQR9, because 9 of 10 of its ligands were amino acids (Table 3). Amino acid sequence alignments showed that the identities of McpCLBD between SQR9 and OI1085 were $61.63 \%$.

More than 140 compounds have been found to induce chemotaxis in Pseudomonas strains (Sampedro et al. 2015), which made these strains models for establishing chemoreceptor

Table 3. Summary of the corresponding ligands of the six chemoreceptors with an extracellular ligand-binding domain region in Bacillus amyloliquefaciens SQR9 and comparison with B. subtilis OI1085 ${ }^{\mathrm{a}}$

\begin{tabular}{|c|c|c|c|c|}
\hline MCP & Ligands $^{\mathbf{b}}$ & Category & Compounds $^{c}$ & In B. subtilis ${ }^{\mathrm{d}}$ \\
\hline \multirow[t]{4}{*}{ McpA } & Dominant & Amino acids & Aspartic acid ${ }^{*}$, glutamic acid ${ }^{*}$, isoleucine ${ }^{*}$, lysine $e^{*}$, tyrosine ${ }^{*}$ & Glucose (r), $\alpha$-methylglucoside (r) \\
\hline & & Acids & $\begin{array}{l}\text { Phthalic acid*, citric acid*, oxalic acid*, malic acid, } \\
\text { succinic acid, fumaric acid, adipic acid, dehydroascorbic acid*, } \\
\text { glyceric acid, 3-hydroxypropionic acid }\end{array}$ & $\cdots$ \\
\hline & & Others & 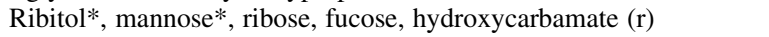 & $\ldots$ \\
\hline & Involved & & Sodium decanoate (r), serine, gluconic acid, fructose & $\ldots$ \\
\hline \multirow[t]{3}{*}{ McpB } & Dominant & Amino acids & Glycine*, tryptophan*, asparagine*, glutamine* & $\begin{array}{l}\text { Asparagine, aspirate, glutamine, } \\
\text { glutamate, and histidine }\end{array}$ \\
\hline & & Acids & Salicylic acid (r), sodium decanoate (r) & $\ldots$ \\
\hline & Involved & & $\begin{array}{l}\text { Adipic acid, ribose, glyceric acid, 3-hydroxypropionic acid, } \\
\text { serine, cystine, methionine, gluconic acid, fructose }\end{array}$ & $\cdots$ \\
\hline \multirow[t]{3}{*}{ McpC } & Dominant & Amino acids & $\begin{array}{l}\text { Valine }{ }^{*}, \text { alanine } *, \text { proline } *, \text { leucine }{ }^{*} \text {, histidine }{ }^{*}, \text { serine, } \\
\text { threonine, cystine, methionine }\end{array}$ & $\begin{array}{l}\text { All amino acids except asparagine, } \\
\text { tryptophan, and histidine }\end{array}$ \\
\hline & & Acids & Gluconic acid & $\ldots$ \\
\hline & Involved & & Succinic acid, maltose & $\ldots$ \\
\hline \multirow[t]{2}{*}{ TlpA } & Dominant & & DL-dithiothreitol* (r) & Unknown \\
\hline & Involved & & Hydroxycarbamate $(r)$, sodium decanoate $(r)$, gluconic acid, maltose & $\ldots$ \\
\hline \multirow[t]{4}{*}{ TlpB } & Dominant & Amino acids & Phenylalanine* & Unknown \\
\hline & & Acids & Pentadecanoic acid* (r) & $\ldots$ \\
\hline & & Others & Dulcitol $^{*}$, inosine*, maltose, fructose & $\ldots$ \\
\hline & Involved & & $\begin{array}{l}\text { Malic acid, succinic acid, fumaric acid, ribose, fucose, } \\
\text { salicylic acid (r), sodium decanoate (r), threonine, gluconic acid }\end{array}$ & $\cdots$ \\
\hline \multirow[t]{2}{*}{ McpR } & Dominant & Amino acids & Arginine* & ND \\
\hline & Involved & & Succinic acid, fucose & $\ldots$ \\
\hline
\end{tabular}

a (r) indicates that this compound is chemorepellent and ND means that this methyl-accepting chemotaxis protein (MCP) is absent in B. subtilis OI1085.

b Ligands information. "Dominant" indicates that this chemoreceptor predominantly mediates the chemotaxis of SQR9 to these compounds, while "involved" means that the MCP is partially involved in chemotaxis to these chemicals.

c Asterisk (*) indicates that these compounds were only sensed by the single chemoreceptor.

${ }^{\mathrm{d}}$ Ligands identified for those orthologous proteins in B. subtilis OI1085 according to the previous literature. 
structure-function relationships (Kato et al. 2008). García et al (2015) reported that McpP of $P$. putida KT2440 had a CACHE-type LBD and mediated taxis to some $\mathrm{C}_{2}$ and $\mathrm{C}_{3}$ carboxylic acids. In this study, the large-scale identification of chemoattractants in root exudates and their responsible chemoreceptors in SQR9 facilitated revealing the chemoreceptor structure-function relationship. Among the 20 ligands of McpA, nine compounds had carboxyl groups at both ends of the chains, which included two amino acids (aspartic acid and glutamic acid) and seven acids (citric acid, malic acid, oxalic acid, fumaric acid, succinic acid, phthalic acid, and adipic acid) (Supplementary Fig. S3). McpA had a dCache domain, which was different from the CACHE domain of McpP in P. putida KT2440. In SQR9, McpC predominantly mediated the chemotaxis to aliphatic amino acids (leucine, alanine, and valine), sulfurbearing amino acids (cystine and methionine), and hydroxy amino acids (threonine and serine), and $\mathrm{McpB}$ seemed to mediate the chemotaxis to the only two amide amino acids, asparagine and glutamine. These results suggest that compounds with similar structure may be sensed by the same chemoreceptor.

Compounds that are attractants for some bacteria may be repellents for others. For example, acetate is an attractant for
Rhodobacter sphaeroides but a repellent for E. coli (Packer and Armitage 2000; Tso and Adler 1974). Interestingly, a compound can switch from being an attractant at a low oxygen concentration to a repellent at atmospheric oxygen concentrations, depending on the growth conditions and the needs of the $R$. sphaeroides cell (Romagnoli et al. 2002). In our work, though $1 \mathrm{mM}$ concentration of the tested ligands in the chemotaxis assay was higher than their actual concentration in the rhizosphere, the SlipChip device allowed the formation of diffusion concentration gradients of the chemoeffector, which mimics the natural condition that plant-released root exudates form a nutrient gradient with distance (Shen et al. 2014). Under this condition, a substantial amount of the tested root exudate components were sensed by SQR9 as chemoattractants, while only a few compounds were chemorepellents. These results reinforce the idea that many PGPR strains show strong chemotactic responses to root exudates, which are very important for the beneficial functions of PGPR.

ITC analysis in vitro is an important approach for verifying whether the binding between chemoreceptor and ligand is direct or not. In this study, the dominant objectives were focused on identification of the MCP mediating chemotactic response of SQR9 to the 39 chemoattractants and 5 chemorepellents by

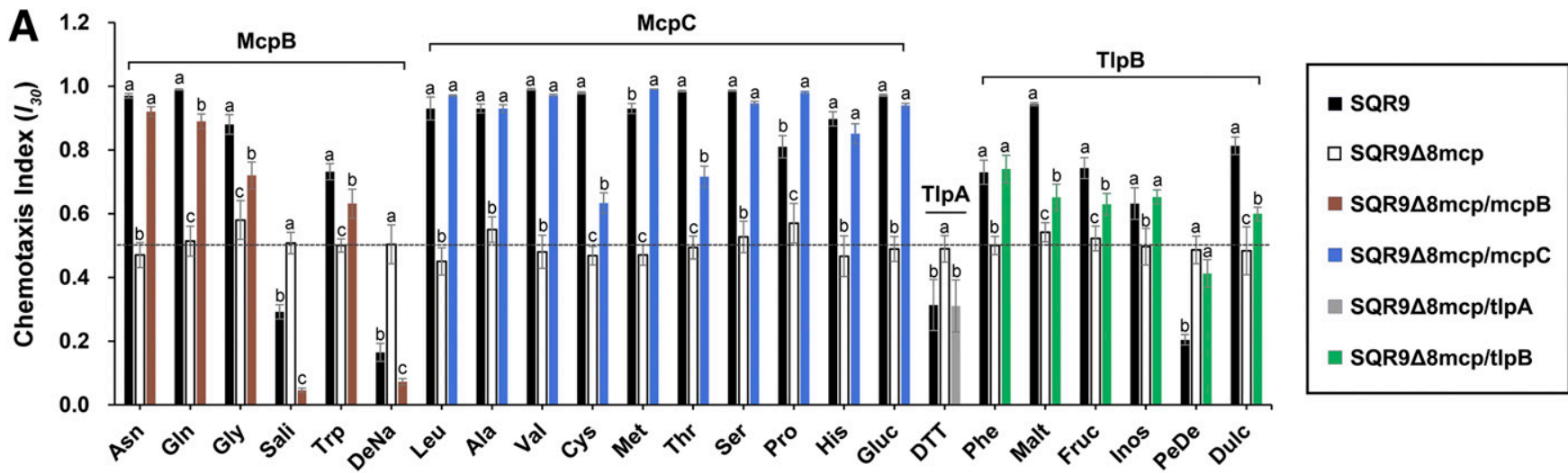

B
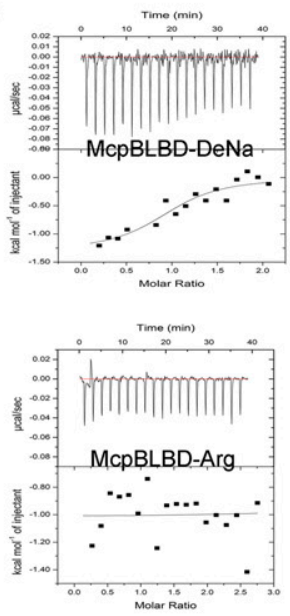
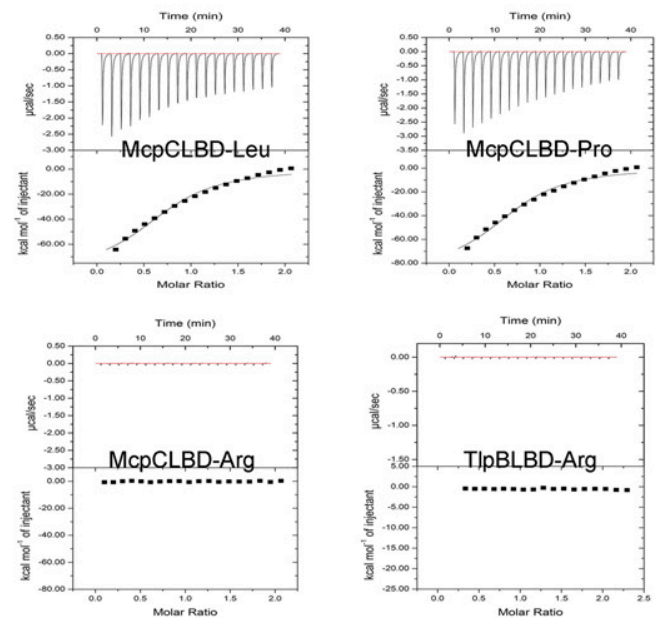
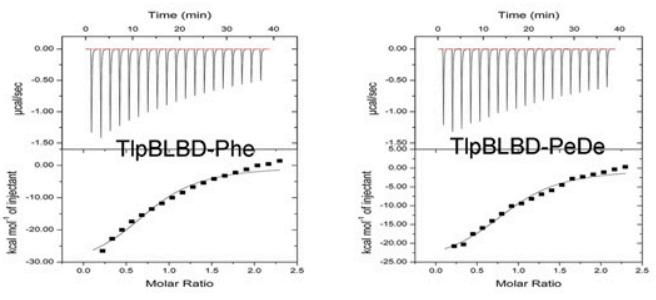

Fig. 3. Chemotaxis activity mediated by the McpB, McpC, TlpA, and TlpB chemoreceptors. A, Chemotactic responses of SQR9, SQR9 $8 m c p$, SQR9 $\Delta 8 m c p / m c p B$, SQR9 $\Delta 8 m c p / m c p C$, SQR9 $\Delta 8 m c p / t l p A$, and SQR9 $\Delta 8 m c p / t l p B$ to 23 compounds. The black dotted line represents a chemotaxis index of 0.5 . The concentration of all the compounds was $1 \mathrm{mM}$. Data for each strain are the means of 13 biological replicates. Error bars represent the standard error of 13 biological replicates. Different letters above the bars indicate significant differences between the three strains for each compound $(P \leq 0.05)$. B, Titration of $20 \mu \mathrm{M}$ McpBLBD, $20.5 \mu \mathrm{M}$ McpCLBD, and $18 \mu \mathrm{M}$ TlpBLBD with $200 \mu \mathrm{M}$ sodium decanoate, leucine, proline, phenylalanine, pentadecanoic acid, and arginine (as a negative control). Upper panels contain the raw titration data and the lower panels show the integrated and dilution-corrected peak areas of the raw data. Data were fitted with the "one binding site model" of the MicroCal version of ORIGIN. Abbreviations: Asn = asparagine, Gln = glutamine, Gly =

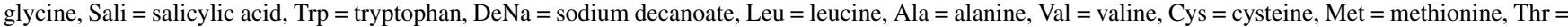
threonine, Ser $=$ serine, Pro $=$ proline , His $=$ histidine, Gluc $=$ gluconic acid, DTT $=$ dl-dithiothreitol, Phe $=$ phenylalanine, Malt $=$ maltose, Fruc $=$ fructose Inos $=$ inosine, PeDe $=$ pentadecanoic acid, and Dulc $=$ dulcitol. 
direct binding or other ways. To explore the correspondence between these ligands and their potential MCP, we randomly picked eight representative compounds to perform the ITC analysis, and excitedly found that all of them can directly bind to the identified chemoreceptors in SQR9.

In conclusion, we have identified 44 compounds in root exudates that the plant-beneficial rhizobacterium $B$. amyloliquefaciens SQR9 chemotactically responded to, 39 of which served as attractants to recruit SQR9 to colonize in the rhizosphere. Among the multiple chemoreceptors of the PGPR strains, the receptors mainly responsible for the rootsecreted organic acids and amino acids may play important roles in the rhizosphere chemotaxis mobility of PGPR. These results not only deepened our insights of PGPR-root interaction but also provided useful information to enhance the rhizosphere chemotaxis mobility and colonization of PGPR with

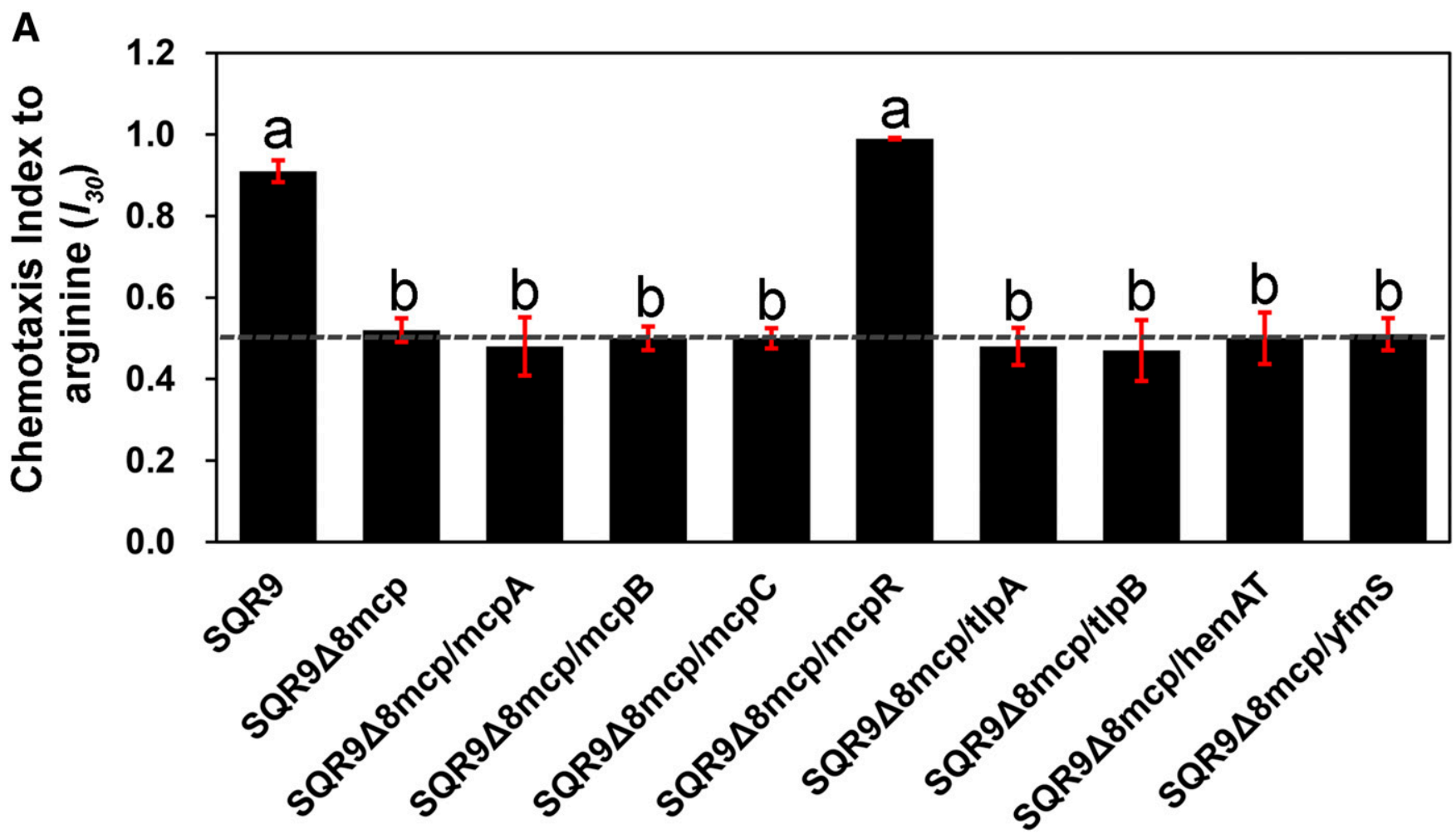

B

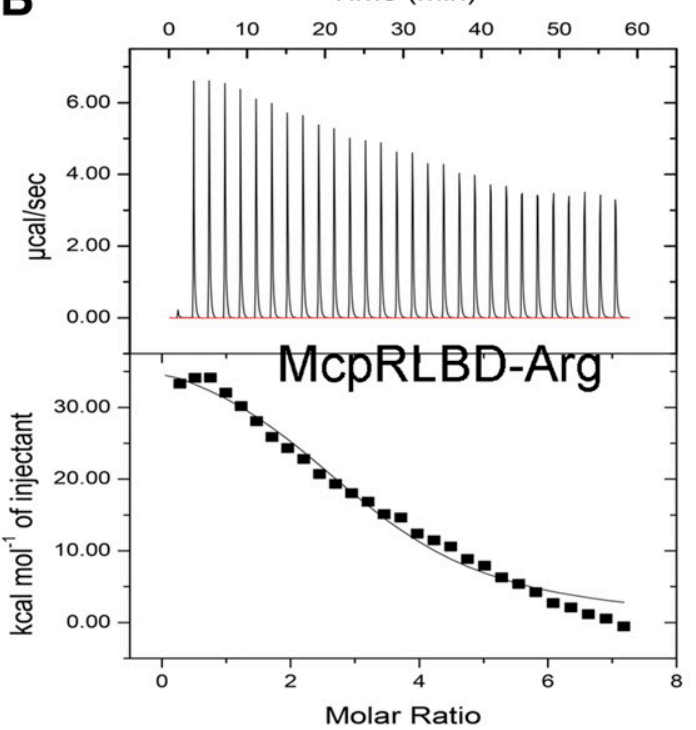

Time (min)

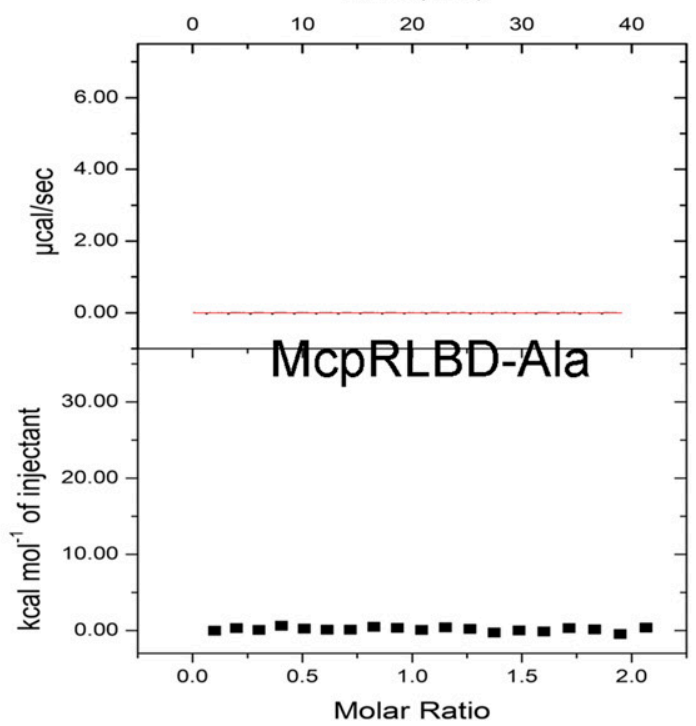

Fig. 4. Chemotaxis index of the different $m c p$ gene complementary strains to arginine and microcalorimetric titration of McpRLBD with arginine. A, Chemotaxis index of the different $m c p$ gene complementary strains to arginine. The black dotted line represents a chemotaxis index of 0.5 . The concentration of arginine was $1 \mathrm{mM}$. Data for each strain are the means of 13 biological replicates. Error bars represent the standard error of 13 biological replicates. Different letters above the bars indicate significant differences $(P \leq 0.05)$. B, Titration of $15 \mu \mathrm{M}$ McpRLBD with $500 \mu \mathrm{M}$ arginine, and alanine (as a negative control). Upper panels contain the titration raw data and the lower panels show the integrated and dilution-corrected peak areas of the raw data. Data were fitted with the "one binding site model" of the MicroCal version of ORIGIN. 
the in vitro addition of a suitable chemoattractant. This approach will increase the efficiency of PGPR in agricultural production and reduce the input of chemical fertilizers and pesticides.

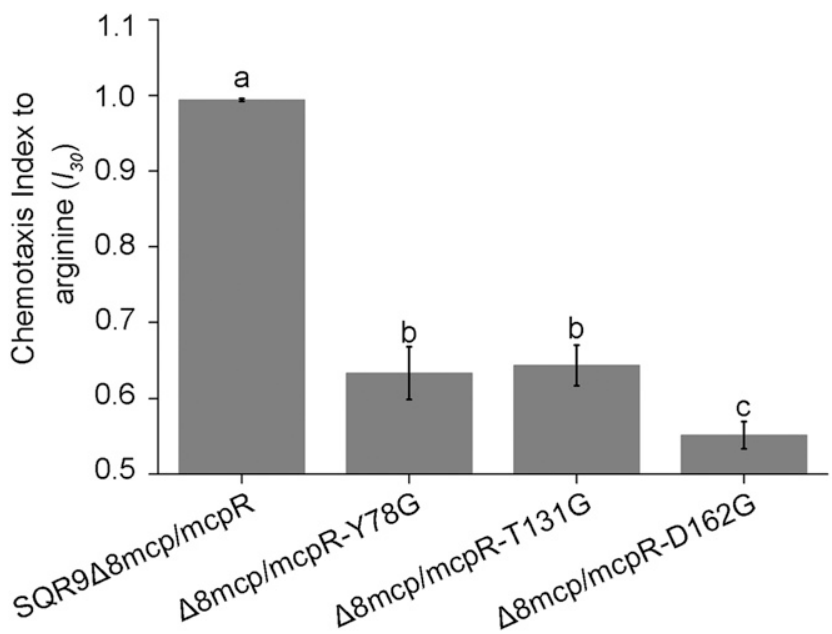

Fig. 5. Chemotactic responses of SQR $9 \Delta 8 m c p$ complemented the wild-type or mutated $m c p R$ to arginine. The concentration of arginine was $1 \mathrm{mM}$. Data for each strain are the means of 13 biological replicates. Error bars represent the standard error of 13 biological replicates. Different letters above the bars indicate significant differences $(P \leq 0.05)$.

\section{MATERIALS AND METHODS}

Bacterial strains, media and growth conditions.

The strains and plasmids used in this study are described in Table 1. B. amyloliquefaciens SQR9 (China General Microbiology Culture Collection Center, CGMCC accession number 5808) was isolated from the cucumber rhizosphere. SQR9 was grown at $37^{\circ} \mathrm{C}$ in low-salt Luria-Bertani (LB) medium (peptone at $10 \mathrm{~g} \mathrm{liter}^{-1}$, yeast extract at $5 \mathrm{~g} \mathrm{liter}^{-1}$, and $\mathrm{NaCl}$ at $3 \mathrm{~g} \mathrm{liter}^{-1}$ ) that was solidified with $15 \mathrm{~g} \mathrm{liter}^{-1}$ agar. E. coli BL21 (DE3) cells were grown at $37^{\circ} \mathrm{C}$ in Luria-Bertani medium (peptone at $10 \mathrm{~g} \mathrm{liter}^{-1}$, yeast extract at $5 \mathrm{~g} \mathrm{liter}^{-1}$, and $\mathrm{NaCl}$ at $5 \mathrm{~g} \mathrm{liter}^{-1}$ ) solidified with agar at $15 \mathrm{~g} \mathrm{liter}^{-1}$. When necessary, the final concentrations of antibiotics were added as follows: chloramphenicol $(\mathrm{Cm})$ at $5 \mathrm{mg}$ liter ${ }^{-1}$, zeocin at $20 \mathrm{mg} \mathrm{liter}^{-1}$, spectinomycin (Spc) at $100 \mathrm{mg} \mathrm{liter}^{-1}$, and kanamycin (Kan) at $30 \mathrm{mg} \mathrm{liter}^{-1}$.

\section{MCP gene prediction and deletion}

in B. amyloliquefaciens SQR9.

Based on the whole genome sequence of B. amyloliquefaciens SQR9 (NCBI accession number CP006890) (Zhang et al. 2015), the potential genes encoding for MCP were predicated according to the standard existence of a MA domain (Alexander and Zhulin 2007).

Considering the functional redundancy of MCP for sensing chemoeffectors as reported in many organisms (Glekas et al. 2012, Ni et al. 2013, Oku et al. 2012, Webb et al. 2014), the $B$. amyloliquefaciens SQR9 mutant deficient in all eight

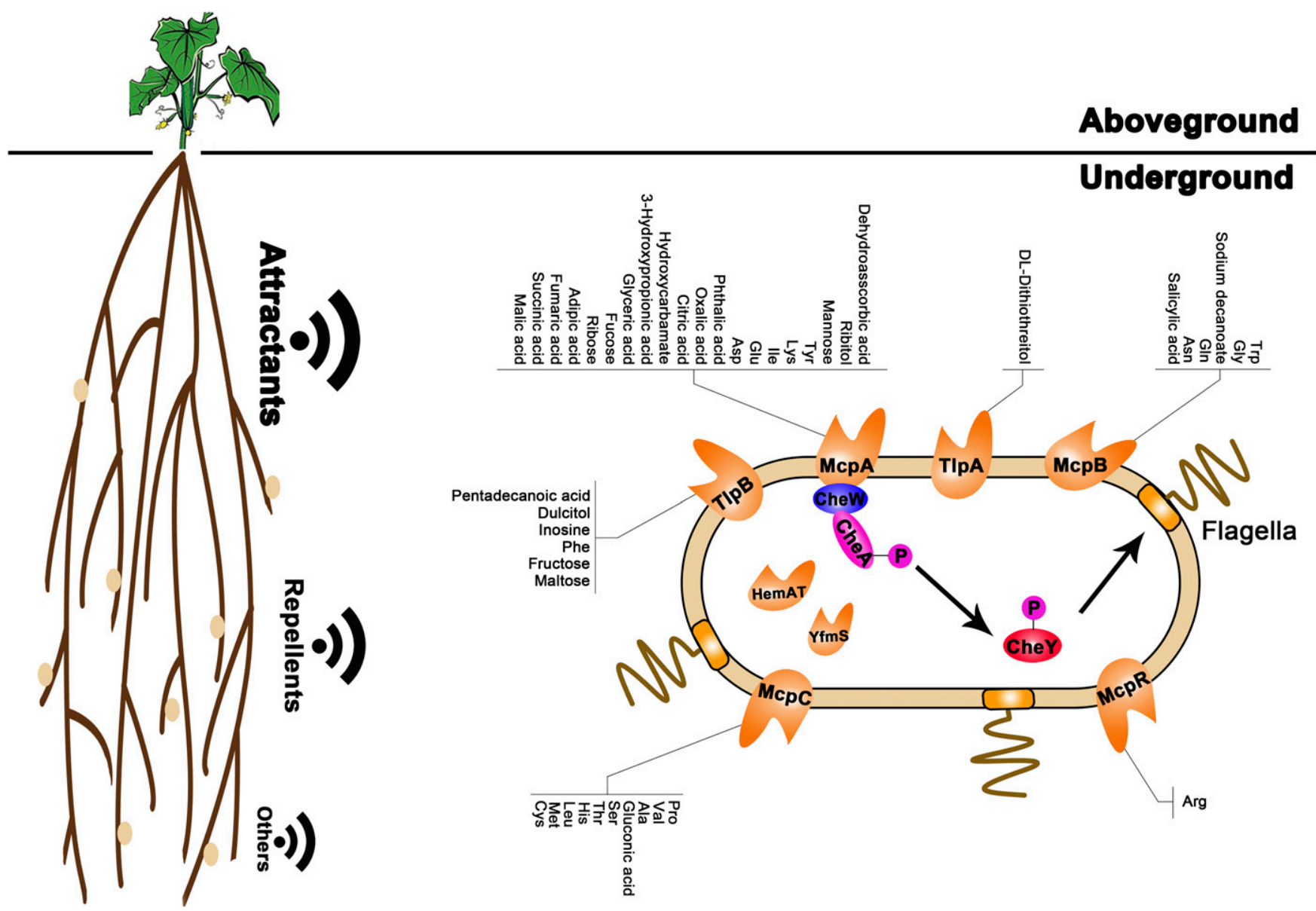

Cucumber

SQR9

Fig. 6. Proposed model of the chemotaxis mobility of Bacillus amyloliquefaciens SQR9 toward cucumber root-secreted compounds mediated by different chemoreceptors. 
predicated mcp genes was constructed using an unmarked genetic manipulation based on multiple gene deletions that rely on a counter-selectable marker, pheS (Zhou et al. 2017). First, four adjacent genes (tlpB-mcpA-tlpA-mcpB, 8,393 bp) were deleted in the first-round knockout to generate SQR9 4 4mcp. Then, mсрC (1,964 bp), mcpR (V529_10510; 1,647 bp), hemAT $(1,290 \mathrm{bp})$, and $y \mathrm{fmS}(852 \mathrm{bp})$ were further deleted one by one in the next rounds of knockout to finally acquire SQR9 $\Delta 8 m c p$ (through SQR9 $5 m c p$, SQR9 $46 m c p$, and SQR9 $\Delta 7 m c p$, respectively). All of these mutants were confirmed by polymerase chain reaction (PCR) and sequencing with the primer sets listed in Supplementary Table S6.

\section{Complementation of the disrupted genes.}

The eight $m c p$ genes were separately integrated into the chromosomal amyE locus of the chemoreceptor-deficient SQR9 $\Delta 8 m c p$ to obtain single- $m c p$-gene complementary strains (SQR9 $48 m c p / m c p A, \mathrm{SQR} 9 \Delta 8 m c p / m c p B$, SQR9 $8 m c p / m c p C$, $\mathrm{SQR} 9 \Delta 8 m c p / t l p A, \mathrm{SQR} 9 \Delta 8 m c p / t l p B, \mathrm{SQR} 9 \Delta 8 m c p / m c p R$, SQR9 $\Delta 8 m c p / h e m A T$, and SQR9 $\Delta 8 m c p / y f m S)$. First, the full sequence of each of the eight $m c p$ genes $(m c p A, m c p B, m c p C$, $t l p A, t l p B, m c p R$, hemAT, and $y f m S$ ) with its native promoter was amplified from SQR9 genomic DNA, which partially overlapped with $\mathrm{Cm}$ or $\mathrm{Spc}$ resistance genes $\left(\mathrm{Cm}^{R}\right.$ or $\left.S p c^{R}\right)$ and the downstream fragments. After that, an upstream $0.70-\mathrm{kb}$ fragment and a downstream $0.74-\mathrm{kb}$ fragment of the $1.97-\mathrm{kb}$ $a m y E$ gene were amplified with the primer set amyE-UF/amyEUR. Subsequently, the $1.0-\mathrm{kb} C m^{R}$ and $S p c^{R}$ genes were amplified from the plasmid pNW33n and p7S6 (Yan et al. 2008) with primer sets $\mathrm{P} 1 / \mathrm{P} 2$ and $\mathrm{P} 3 / \mathrm{P} 4$, respectively, which overlapped with the upstream fragments and the complemented genes. Finally, the obtained four fragments were fused by overlap PCR, and the fused fragments for each of the eight target genes were individually transformed into $\Delta 8 m c p$-competent cells. The obtained transformants were verified using primer sets (Zhou et al. 2017).

\section{Chemotaxis assay.}

The chemotaxis assay was performed as described by Shen et al. (2014) by using a simple and reusable microfluidic SlipChip device. By a simple slipping operation, three microwells were disconnected from other units and interconnected by the ducts, which allowed the formation of diffusion concentration gradients of the chemoeffector for inducing cell migration from the cell microcells toward the other two microwells. As a result, the concentration of the chemoeffector sensed by bacteria was lower than its initial concentration. Briefly, a $10-\mathrm{mg} \mathrm{ml}^{-1}$ bovine serum albumin (BSA) solution was injected into all channels and used to wash the microwells for $5 \mathrm{~min}$. After removing the BSA solution using a vacuum across the access holes, the solutions containing different chemoeffectors, bacterial cells suspended in phosphate-buffered saline (PBS) buffer $\left(\mathrm{NaCl}\right.$ at $8 \mathrm{~g} \mathrm{liter}^{-1}, \mathrm{KCl}$ at $0.2 \mathrm{~g} \mathrm{liter}^{-1}, \mathrm{Na}_{2} \mathrm{HPO}_{4}$ at $1.44 \mathrm{~g}$ liter ${ }^{-1}$, and $\mathrm{KH}_{2} \mathrm{PO}_{4}$ at $0.24 \mathrm{~g} \mathrm{liter}^{-1}$; $\mathrm{pH}$ 7.4), and PBS buffer alone (negative control) were loaded to the top, middle, and bottom microwells, respectively. Subsequently, the SlipChip device was slipped to ensure that the cells could migrate freely from the middle microwells to the ducts and the microwells were loaded with the chemoeffector or PBS. The device was placed on an inverted fluorescence microscope (Ti-Eclipse; Nikon) and kept for $30 \mathrm{~min}$ in the dark at room temperature. Then, the cells in the top microwells and bottom microwells were monitored. The chemotaxis index $\left(I_{t}\right)$ was used to indicate the chemotactic ability of the bacterial cells with a chemoeffector at a certain concentration. $I_{t}$ was defined as $N_{e} /\left(N_{e}+N_{c}\right)$, where $N_{e}$ is the number of cells that migrated into the chemoeffectorcontaining microwells and $N_{c}$ is the number of cells that migrated into the control microwells in a certain time period.
In this study, $I_{30}$ was used to characterize chemotaxis; cells were allowed to migrate freely in the gradient for $30 \mathrm{~min}$. In theory, an $I_{30}$ value approximately equal to 0.5 means that the cells do not respond to the chemoeffector, while an $I_{30}$ value of more than 0.5 indicates that the cells are attracted by the chemoeffector and an $I_{30}$ value lower than 0.5 suggests the cells are repelled by the chemoeffector. Here, to ensure the stability and reliability of the assessment, we redefined the nonresponse interval of $I_{30}$ to the range between 0.4 and 0.6 , while $>0.6$ and $<0.4$ were defined as positive and negative responses to the chemoeffector, respectively.

\section{Protein expression and purification.}

Expression and purification of the LBD of McpA, McpB, McpC, TlpA, TlpB, and McpR in B. amyloliquefaciens SQR9 were performed. The DNA fragments encoding the LBD of McpA (Ala33-Pro278), McpB (Glu33-Pro278), McpC (Lys33Met274), TlpA (Ala33-Pro278), TlpB (Ala33-Met282), and McpR (Ser35-Gln165) were amplified with primer sets that contained restriction sites for NdeI and HindIII using SQR9 genomic DNA as a template. The PCR products were digested and cloned into the expression plasmid pET28a(+), which was linearized with the NdeI and HindIII enzymes. The insert and flanking regions of the six reconstructed plasmids (pET28aMcpALBD, pET28a-McpBLBD, pET28a-McpCLBD, pET28aTlpALBD, pET28a-TlpBLBD, and pET28a-McpRLBD) were verified by DNA sequencing. The expressed proteins contained an N-terminal His tag.

The six constructed expression vectors were individually introduced into $E$. coli BL21 (DE3). The obtained strains were grown in 1-liter Erlenmeyer flasks containing $200 \mathrm{ml}$ of LB medium supplemented with Kan at $30 \mathrm{mg} \mathrm{liter}^{-1}$ at $37^{\circ} \mathrm{C}$, with shaking at $220 \mathrm{rpm}$. When the optical density at $600 \mathrm{~nm}$ of the culture reached 0.6, the growth temperature was lowered to $16^{\circ} \mathrm{C}$. After another 30 -min incubation, the protein expressions were induced by adding $0.05 \mathrm{mM}$ isopropyl- $\beta$-Dthiogalactopyranoside. The growth was continued at $16^{\circ} \mathrm{C}$, with shaking at $220 \mathrm{rpm}$ overnight, prior to cell harvest by centrifugation at $8,000 \times g$ for $10 \mathrm{~min}$. Then, the cells were washed at least twice with $0.01 \mathrm{M}$ PBS. The cell pellets were resuspended in $50 \mathrm{ml}$ of $0.01 \mathrm{M}$ PBS and disrupted by sonication (2-s pulse on, 3-s pulse off). The cellular lysates were centrifuged at $20,000 \times g$ for $50 \mathrm{~min}$. Finally, for further clarification, the lysates were passed through a $0.22-\mu \mathrm{m}$ filter (Millipore) to remove any other aggregates or insoluble particles, followed by purification with His-affinity resin chromatography according to the manufacturer's (Hua Chun) instructions. All the purified proteins were collected and stored in TKMDmod buffer $\left(50 \mathrm{mM}\right.$ Tris, $\mathrm{pH} 8.0 ; 50 \mathrm{mM} \mathrm{KCl}$; and $5 \mathrm{mM} \mathrm{MgCl}_{2}$ ) at $-80^{\circ} \mathrm{C}$.

\section{Isothermal titration calorimetry.}

All ITC measurements were performed using an iTC200 titration calorimeter (MicroCal; GE) at $25^{\circ} \mathrm{C}$, and the data were extracted and processed using the Origin software package. All of the ligands were dissolved in TKMDmod buffer. For ligand binding analysis, the protein was placed into the sample cell and was titrated with aliquots of ligand solution in the injector syringe. The $400-\mu l$ reaction cell was constantly stirred at $750 \mathrm{rpm}$. The mean enthalpies measured from the injection of the ligands into the buffer were subtracted from raw titration data prior to data analysis using the "one binding site model" of the MicroCal version of ORIGIN. The two important constants, $K_{D}$ and $\Delta H$, were gained. The smaller the $K_{D}$ value, the stronger the binding of the protein and ligand. Moreover, the binding is endothermic when the $\Delta H$ is greater than zero. When the $\Delta H$ is less than zero, the binding is exothermic. 


\section{ACKNOWLEDGMENTS}

We thank J. Luo (Nanjing University) for help in ITC experiments, D. Chen (Chinese Academy of Sciences) for the help in chemotaxis assay, and Y. Miao (Nanjing Agricultural University) for excellent technical assistance.

\section{LITERATURE CITED}

Alexander, R. P., and Zhulin, I. B. 2007. Evolutionary genomics reveals conserved structural determinants of signaling and adaptation in microbial chemoreceptors. Proc. Natl. Acad. Sci. USA 104:2885-2890.

Allard-Massicotte, R., Tessier, L., Lecuyer, F., Lakshmanan, V., Lucier, J. F., and Garneau, D. 2016. Bacillus subtilis early colonization of Arabidopsis thaliana roots involves multiple chemotaxis receptors. MBio 7:e01664-16.

Aravind, L., and Ponting, C. P. 1999. The cytoplasmic helical linker domain of receptor histidine kinase and methyl-accepting proteins is common to many prokaryotic signalling proteins. FEMS Microbiol. Lett. 176:111-116.

Badri, D. V., and Vivanco, J. M. 2009. Regulation and function of root exudates. Plant Cell Environ. 32:666-681.

Bais, H. P., Weir, T. L., Perry, L. G., Gilroy, S., and Vivanco, J. M. 2006. The role of root exudates in rhizosphere interactions with plants and other organisms. Annu. Rev. Plant Biol. 57:233-266.

Baker, M. D., Wolanin, P. M., and Stock, J. B. 2006. Signal transduction in bacterial chemotaxis. BioEssays 28:9-22.

Berendsen, R. L., Pieterse, C. M., and Bakker, P. A. 2012. The rhizosphere microbiome and plant health. Trends Plant Sci. 17:478-486.

Caetano-Anollés, G., Wall, L. G., De Micheli, A. T., Macchi, E. M., Bauer W. D., and Favelukes, G. 1988. Role of motility and chemotaxis in efficiency of nodulation by Rhizobium meliloti. Plant Physiol. 86: 1228-1235.

Cao, Y., Zhang, Z., Ling, N., Yuan, Y., Zheng, X., and Shen, B. 2011. Bacillus subtilis SQR9 can control Fusarium wilt in cucumber by colonizing plant roots. Biol. Fertil. Soils 47:495-506.

Compant, S., Clément, C., and Sessitsch, A. 2010. Plant growth-promoting bacteria in the rhizo- and endosphere of plants: Their role, colonization, mechanisms involved and prospects for utilization. Soil Biol. Biochem. 42:669-678.

de Weert, S., Vermeiren, H., Mulders, I. H. M., Kuiper, I., Hendrickx, N., Bloemberg, G. V., Vanderleyden, J., De Mot, R., and Lugtenberg, B. J. J. 2002. Flagella-driven chemotaxis towards exudate components is an important trait for tomato root colonization by Pseudomonas fluorescens. Mol. Plant-Microbe Interact. 15:1173-1180.

Fan, B., Blom, J., Klenk, H. P., and Borriss, R. 2017. Bacillus amyloliquefaciens, Bacillus velezensis, and Bacillus siamensis form an "operational group B. amyloliquefaciens" within the B. subtilis species complex. Front. Microbiol. 8:22.

García, V., Reyes-Darias, J. A., Martin-Mora, D., Morel, B., Matilla, M. A., and Krell, T. 2015. Identification of a chemoreceptor for C2 and C3 carboxylic acids. Appl. Environ. Microbiol. 81:5449-5457.

Garrity, L. F., and Ordal, G. W. 1995. Chemotaxis in Bacillus subtilis: How bacteria monitor environmental signals. Pharmacol. Ther. 68:87-104.

Glekas, G. D., Foster, R. M., Cates, J. R., Estrella, J. A., Wawrzyniak, M. J., and Rao, C. V. 2010. A PAS domain binds asparagine in the chemotaxis receptor McpB in Bacillus subtilis. J. Biol. Chem. 285:1870-1878.

Glekas, G. D., Mulhern, B. J., Kroc, A., Duelfer, K. A., Lei, V., and Rao, C. V. 2012. The Bacillus subtilis chemoreceptor McpC senses multiple ligands using two discrete mechanisms. J. Biol. Chem. 287:39412-39418.

Hanlon, D. W., and Ordal, G. W. 1994. Cloning and characterization of genes encoding methyl-accepting chemotaxis proteins in Bacillus subtilis. J. Biol. Chem. 269:14038-14046.

Hou, S., Larsen, R. W., Boudko, D., Riley, C. W., Karatan, E., and Zimmer, M. 2000. Myoglobin-like aerotaxis transducers in Archaea and Bacteria. Nature 403:540-544.

Jiang, N., Liu, W., Li, Y., Wu, H., Zhang, Z., and Alexandre, G. 2016. A chemotaxis receptor modulates nodulation during the Azorhizobium caulinodans-Sesbania rostrata symbiosis. Appl. Environ. Microbiol. 82: 3174-3184.

Kato, J., Kim, H. E., Takiguchi, N., Kuroda, A., and Ohtake, H. 2008. Pseudomonas aeruginosa as a model microorganism for investigation of chemotactic behaviors in ecosystem. J. Biosci. Bioeng. 106:1-7.

Lacal, J., Garcia-Fontana, C., Munoz-Martinez, F., Ramos, J. L., and Krell, T. 2010. Sensing of environmental signals: Classification of chemoreceptors according to the size of their ligand binding regions. Environ. Microbiol. 12:2873-2884.

Liu, Y., Chen, L., Wu, G., Feng, H., Zhang, G., and Shen, Q. 2017. Identification of root-secreted compounds involved in the communication between cucumber, the beneficial Bacillus amyloliquefaciens, and the soil-borne pathogen Fusarium oxysporum. Mol. Plant-Microbe Interact. 30:53-62.

Lugtenberg, B., and Kamilova, F. 2009. Plant-growth-promoting rhizobacteria. Annu. Rev. Microbiol. 63:541-556.

Milburn, M. V., Privé, G. G., Milligan, D. L., Scott, W. G., Yeh, J., and Jancarik, J. 1991. Three-dimensional structures of the ligand-binding domain of the bacterial aspartate receptor with and without a ligand Science 254:1342-1347.

Mowbray, S. L., and Sandgren, M. O. 1998. Chemotaxis receptors: A progress report on structure and function. J. Struct. Biol. 124:257-275.

Ni, B., Huang, Z., Fan, Z., Jiang, C. Y., and Liu, S. J. 2013. Comamonas testosteroni uses a chemoreceptor for tricarboxylic acid cycle intermediates to trigger chemotactic responses towards aromatic compounds. Mol. Microbiol. 90:813-823.

Oku, S., Komatsu, A., Tajima, T., Nakashimada, Y., and Kato, J. 2012 Identification of chemotaxis sensory proteins for amino acids in Pseudomonas fluorescens $\mathrm{Pf0}-1$ and their involvement in chemotaxis to tomato root exudate and root colonization. Microbes Environ. 27:462-469.

Packer, H. L., and Armitage, J. P. 2000. Behavioral responses of Rhodobacter sphaeroides to linear gradients of the nutrients succinate and acetate. Appl. Environ. Microbiol. 66:5186-5191.

Porter, S. L., Wadhams, G. H., and Armitage, J. P. 2011. Signal processing in complex chemotaxis pathways. Nat. Rev. Microbiol. 9:153-165.

Rico-Jiménez, M., Muñoz-Martínez, F., García-Fontana, C., Fernandez, M., Morel, B., and Ortega, A. 2013. Paralogous chemoreceptors mediate chemotaxis towards protein amino acids and the non-protein amino acid gamma-aminobutyrate (GABA). Mol. Microbiol. 88:1230-1243.

Romagnoli, S., Packer, H. L., and Armitage, J. P. 2002. Tactic responses to oxygen in the phototrophic bacterium Rhodobacter sphaeroides WS8N. J. Bacteriol. 184:5590-5598

Sampedro, I., Parales, R. E., Krell, T., and Hill, J. E. 2015. Pseudomonas chemotaxis. FEMS Microbiol. Rev. 39:17-46.

Scharf, B. E., Hynes, M. F., and Alexandre, G. M. 2016. Chemotaxis signaling systems in model beneficial plant-bacteria associations. Plant Mol. Biol. 90:549-559.

Schultz, J., Milpetz, F., Bork, P., and Ponting, C. P. 1998. SMART, a simple modular architecture research tool: Identification of signaling domains. Proc. Natl. Acad. Sci. USA 95:5857-5864.

Shen, C., Xu, P., Huang, Z., Cai, D., Liu, S. J., and Du, W. 2014. Bacterial chemotaxis on slipChip. Lab Chip 14:3074-3080.

Sourjik, V. 2004. Receptor clustering and signal processing in E. coli chemotaxis. Trends Microbiol. 12:569-576.

Sourjik, V., and Wingreen, N. S. 2012. Responding to chemical gradients: Bacterial chemotaxis. Curr. Opin. Cell Biol. 24:262-268.

Szurmant, H., and Ordal, G. W. 2004. Diversity in chemotaxis mechanisms among the bacteria and archaea. Microbiol. Mol. Biol. Rev. 68:301-319.

Tso, W. W., and Adler, J. 1974. Negative chemotaxis in Escherichia coli. J. Bacteriol. 118:560-576.

Webb, B. A., Hildreth, S., Helm, R. F., and Scharf, B. E. 2014 Sinorhizobium meliloti chemoreceptor McpU mediates chemotaxis toward host plant exudates through direct proline sensing. Appl. Environ. Microbiol. 80:3404-3415.

Webb, B. A., Karl Compton, K., Castaneda Saldana, R., Arapov, T. D., Keith Ray, W., and Helm, R. F. 2017. Sinorhizobium meliloti chemotaxis to quaternary ammonium compounds is mediated by the chemoreceptor McpX. Mol. Microbiol. 103:333-346.

Weng, J., Wang, Y., Li, J., Shen, Q., and Zhang, R. 2013. Enhanced root colonization and biocontrol activity of Bacillus amyloliquefaciens SQR9 by $a b r B$ gene disruption. Appl. Microbiol. Biotechnol. 97: 8823-8830.

Yan, X., Yu, H. J., Hong, Q., and Li, S. P. 2008. Cre/lox system and PCRbased genome engineering in Bacillus subtilis. Appl. Environ. Microbiol. 74:5556-5562

Yssel, A., Reva, O., and Tastan Bishop, O. 2011. Comparative structural bioinformatics analysis of Bacillus amyloliquefaciens chemotaxis proteins within Bacillus subtilis group. Appl. Microbiol. Biotechnol. 92:997-1008.

Zhang, N., Wang, D., Liu, Y., Li, S., Shen, Q., and Zhang, R. 2014. Effects of different plant root exudates and their organic acid components on chemotaxis, biofilm formation and colonization by beneficial rhizosphere-associated bacterial strains. Plant Soil 374:689-700.

Zhang, N., Yang, D., Wang, D., Miao, Y., Shao, J., and Zhou, X. 2015. Whole transcriptomic analysis of the plant-beneficial rhizobacterium Bacillus amyloliquefaciens SQR9 during enhanced biofilm formation regulated by maize root exudates. BMC Genomics 16:685.

Zhou, C., Shi, L., Ye, B., Feng, H., Zhang, J., and Zhang, R. 2017. PheS*, an effective host-genotype-independent counter-selectable marker for marker-free chromosome deletion in Bacillus amyloliquefaciens. Appl. Microbiol. Biotechnol. 101:217-227. 